\title{
Structure of $\boldsymbol{R}$-(1-Naphtyl)ethylamine Hydrochloride Monohydrate
}

\author{
Isao FUJII and Noriaki HiraYAMA \\ Department of Biological Science and Technology, Tokai University, \\ Nishino, Numazu, Shizuoka 410-0321, Japan
}

(Received May 30, 2000; Accepted June 26, 2000)

The title compound (Fig. 1), R-NPEA, is one of the useful resolving agents of chiral acids. The degree of chiral discrimination and the efficiency of the optical agents are considered to be dependent on the three-dimensional structures of the agents. In order to understand the mechanism of chiral recognition of $R$-NPEA, the inherent three-dimensional structure of this useful optical selector was determined by X-ray analysis.

The gelation of a chloroform solution of $R$-NPEA $(1 \% \mathrm{v} / \mathrm{v})$ takes place by diffusing hydrogen chloride into the solution. The gel was dissolved in ethanol and colorless rod-like crystals were obtained from the solution at $297(2) \mathrm{K}$. A crystal with dimensions of $0.30 \times 0.20 \times 0.20 \mathrm{~mm}$ was used for diffraction experiments. The crystal and experimental data are given in Table 1. The structure was solved by direct methods and<smiles>C[C@H](N)c1cccc2ccccc12</smiles>

Fig. 1 Chemical structure.

Table 1 Crystal and experimental data

$$
\begin{aligned}
& \text { Formula: } \mathrm{C}_{12} \mathrm{H}_{16} \mathrm{NOCl} \\
& \text { Formula weight }=225.72 \\
& \text { Crystal system: monoclinic } \\
& \text { Space group: } P 2_{1} \\
& a=10.6050(7) \AA \\
& b=7.3924(4) \AA \\
& c=8.0816(4) \AA \\
& V=616.08(6) \AA^{3} \\
& D_{\mathrm{x}}=1.217 \mathrm{Mg} \mathrm{\textrm {m } ^ { - 3 }} \\
& \text { No. of reflections used }=2644 \\
& 2 \theta_{\max }=148^{\circ} \text { with Cu } \mathrm{K}_{\alpha} \\
& R=0.041 \\
& (\Delta / \sigma)_{\max }=0.05 \\
& (\Delta \rho)_{\max }=0.43 \\
& (\Delta \rho)_{\min }=-0.28 \\
& \text { Measurement: Enraf-Nonius CAD-4 Turbo } \\
& \text { Program system: Rigaku teXsan }{ }^{1} \\
& \text { Structure determination: SIR } 88^{2} \\
& \text { Refinement: full-matrix }
\end{aligned}
$$

refined with anisotropic temperature factors for non- $\mathrm{H}$ atoms. All $\mathrm{H}$ atoms were found from difference Fourier maps. The $\mathrm{H}$ atoms were refined with isotropic temperature factors. The absolute configuration, determined using anomalous dispersion by chlorine, confirms the $R$ configuration. The atomic parameters for non-hydrogen atoms are listed in Table 2.

An ORTEP II $^{3}$ drawing of the title compound together with the atomic numbering is shown in Fig. 2. The bond distances and angles are tabulated in Table 3. The two phenyl rings are essentially planar. The dihedral angles between the mean planes is $2.82(7)^{\circ}$ and the naphtyl ring is significantly folded across the central $\mathrm{C}-\mathrm{C}$ bond toward the $\mathrm{C} 2$ atom. The ethyl group is almost perpendicular to the naphtyl ring with the torsion angles of $\mathrm{C} 2-\mathrm{C} 1-\mathrm{C} 3-\mathrm{C} 10 \mathrm{a}$ and $\mathrm{N} 1-\mathrm{C} 1-\mathrm{C} 3-\mathrm{C} 4$ being $79.3(2)$ and $24.0(2)^{\circ}$, respectively. The C6a-C10a bond is significantly longer than other bonds in the naphtyl ring. The shortest bond in the ring is the C5-C6 bond. The C6a-C10a-C10 and C6a-C10a-C3 bond angles are significantly smaller than the others inside the naphtyl ring. The exocyclic bond angles around the $\mathrm{C} 3$ atom are highly asymmetrical. $R$ NPEA molecules, chloride ions and water molecules form an extensive hydrogen bond network in the crystal. $R$-NPEA molecules are connected through chloride ions and water molecules. There are four intermolecular hydrogen bonds $\left[\mathrm{O}-\mathrm{H} \cdots \mathrm{Cl}=3.257(2), \angle \mathrm{O}-\mathrm{H} \cdots \mathrm{Cl}=168(4)^{\circ} ; \mathrm{O}-\mathrm{H} \cdots \mathrm{Cl}(2-x\right.$,

Table 2 Atomic coordinates and equivalent isotropic thermal parameters $\left(B_{\text {eq }}\right)$

\begin{tabular}{lllll}
\hline Atom & $x$ & $y$ & $z$ & $B_{\mathrm{eq}}$ \\
\hline $\mathrm{C} 1$ & $0.00573(4)$ & 0 & $0.74582(6)$ & $3.330(9)$ \\
$\mathrm{O}$ & $0.0456(2)$ & $0.1765(3)$ & $1.1475(2)$ & $4.06(4)$ \\
$\mathrm{N}$ & $0.0881(2)$ & $0.4748(3)$ & $0.6589(2)$ & $2.73(3)$ \\
$\mathrm{C} 1$ & $0.2290(2)$ & $0.5248(2)$ & $0.7188(2)$ & $2.42(3)$ \\
$\mathrm{C} 2$ & $0.2493(2)$ & $0.7090(3)$ & $0.6462(3)$ & $3.10(4)$ \\
$\mathrm{C} 3$ & $0.3154(2)$ & $0.3793(2)$ & $0.6716(2)$ & $2.33(3)$ \\
$\mathrm{C} 4$ & $0.2702(2)$ & $0.2635(3)$ & $0.5384(2)$ & $2.91(4)$ \\
$\mathrm{C} 5$ & $0.3510(2)$ & $0.1291(3)$ & $0.4928(3)$ & $3.45(4)$ \\
$\mathrm{C} 6$ & $0.4759(2)$ & $0.1137(3)$ & $0.5805(3)$ & $3.29(4)$ \\
$\mathrm{C} 6 \mathrm{a}$ & $0.5287(2)$ & $0.2334(3)$ & $0.7153(2)$ & $2.62(3)$ \\
$\mathrm{C} 7$ & $0.6614(2)$ & $0.2254(3)$ & $0.8007(3)$ & $3.29(4)$ \\
$\mathrm{C} 8$ & $0.7132(2)$ & $0.3447(4)$ & $0.9267(3)$ & $3.60(4)$ \\
$\mathrm{C} 9$ & $0.6337(2)$ & $0.4764(4)$ & $0.9772(3)$ & $3.66(4)$ \\
$\mathrm{C} 10$ & $0.5047(2)$ & $0.4868(3)$ & $0.8984(2)$ & $3.05(4)$ \\
$\mathrm{C} 10 \mathrm{a}$ & $0.4477(2)$ & $0.3686(2)$ & $0.7635(2)$ & $2.35(3)$ \\
\hline
\end{tabular}

$B_{\text {eq }}=(4 / 3) \sum_{i} \Sigma_{j} \beta_{i j}\left(\boldsymbol{a}_{i} \cdot \boldsymbol{a}_{j}\right)$. 


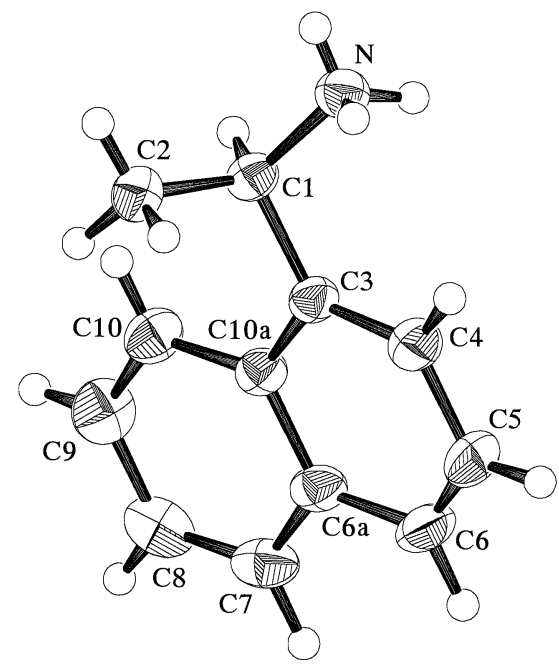

Fig. 2 ORTEPII drawing of the molecule, representing heavy atoms as $50 \%$ probability ellipsoids and $\mathrm{H}$ atoms as spheres of arbitrary radius.

$1 / 2+y, 1-z)=3.172(2), \quad \angle \mathrm{O}-\mathrm{H} \cdots \mathrm{Cl}=157(3)^{\circ} ; \quad \mathrm{N}-\mathrm{H} \cdots \mathrm{Cl}=$ $3.186(2), \angle \mathrm{N}-\mathrm{H} \cdots \mathrm{Cl}=165(3)^{\circ} ; \mathrm{N}-\mathrm{H} \cdots \mathrm{Cl}(2-x, 1 / 2+y, 2-z)=$ $\left.3.279(2), \angle \mathrm{N}-\mathrm{H} \cdots \mathrm{Cl}=173(3)^{\circ}\right]$.

\section{References}

1. teXsan, "Single Crystal Structure Analysis Software,
Table 3 Bond distances $(\AA)$ and angles $\left({ }^{\circ}\right)$

\begin{tabular}{|c|c|c|c|c|c|c|c|c|c|c|}
\hline $\mathrm{N}$ & --- & & \multicolumn{2}{|c|}{$1.506(2)$} & $\mathrm{C} 1$ & --- & C2 & & \multicolumn{2}{|c|}{$1.518(3)$} \\
\hline $\mathrm{C} 1$ & $---\quad C 3$ & & \multicolumn{2}{|c|}{$1.518(2)$} & $\mathrm{C} 1$ & --- & $\mathrm{C} 2$ & & \multicolumn{2}{|c|}{$1.518(3)$} \\
\hline C3 & $---\quad \mathrm{C} 1$ & $10 a$ & \multicolumn{2}{|c|}{$1.429(2)$} & $\mathrm{C} 4$ & --- & C5 & & \multicolumn{2}{|c|}{$1.415(3)$} \\
\hline C5 & $---\quad C 6$ & & \multicolumn{2}{|c|}{$1.354(3)$} & c6 & --- & $\mathrm{C} 6$ & & \multicolumn{2}{|c|}{$1.414(3)$} \\
\hline $\mathrm{c} 6 \mathrm{a}$ & $a--c c 7$ & & \multicolumn{2}{|c|}{$1.417(3)$} & $\mathrm{C} 6 \mathrm{a}$ & -- & $\mathrm{C} 1 \mathrm{C}$ & $.0 a$ & \multicolumn{2}{|c|}{$1.431(2)$} \\
\hline C7 & $---\quad c 8$ & & \multicolumn{2}{|c|}{$1.362(4)$} & $\mathrm{C} 8$ & --- & $\mathrm{C} 9$ & & \multicolumn{2}{|c|}{$1.410(4)$} \\
\hline C9 & $---\quad C 1$ & 10 & \multicolumn{2}{|c|}{$1.370(3)$} & $\mathrm{C} 10$ & --- & $\mathrm{C} 1 \mathrm{C}$ & $.0 a$ & \multicolumn{2}{|c|}{$1.417(3)$} \\
\hline $\mathrm{N}$ & $---\mathrm{C} 1$ & --- & $\mathrm{C} 2$ & $108.4(1)$ & $\mathrm{N}$ & $---c$ & $c 1$ & --- & $\mathrm{C} 3$ & $110.9(1)$ \\
\hline $\mathrm{C} 2$ & $---\mathrm{C} 1$ & --- & $\mathrm{C} 3$ & $113.0(1)$ & $\mathrm{C} 1$ & --- & $\mathrm{C} 3$ & -- & $\mathrm{C} 4$ & $121.3(2)$ \\
\hline $\mathrm{C} 1$ & $---\mathrm{C} 3$ & --- & C10a & $119.0(2)$ & $\mathrm{C} 4$ & --- & $\mathrm{C} 3$ & --- & c10a & $119.6(2)$ \\
\hline C3 & $---\mathrm{C} 4$ & --- & C5 & $121.4(2)$ & $\mathrm{C} 4$ & --- & C5 & --- & $\mathrm{C} 6$ & $120.0(2)$ \\
\hline C5 & $---\mathrm{C} 6$ & --- & $\mathrm{c} 6 \mathrm{a}$ & $120.9(2)$ & $\mathrm{C} 6$ & --- & $\mathrm{c} 6 \mathrm{a}$ & --- & $-\mathrm{C} 7$ & $121.2(2)$ \\
\hline $\mathrm{c} 6$ & $---c 6 a$ & --- & $\mathrm{C} 10 \mathrm{a}$ & $119.5(2)$ & C7 & --- & $\mathrm{C} 6 \mathrm{a}$ & -- & $-\mathrm{C} 10 \mathrm{a}$ & $119.2(2)$ \\
\hline $\mathrm{C} 6 \mathrm{a}$ & $\mathrm{a}-\mathrm{-C} 7$ & --- & C8 & $121.2(2)$ & C7 & --- & C8 & --- & C9 & $120.0(2)$ \\
\hline $\mathrm{C} 8$ & $---C 9$ & --- & $\mathrm{C} 10$ & $120.3(2)$ & C9 & --- & $\mathrm{C} 10$ & --- & $-c 10 a$ & $121.5(2)$ \\
\hline C3 & $--\mathrm{C} 10$ & $a--$ & $\mathrm{C} 6 \mathrm{a}$ & $118.5(2)$ & C3 & --- & $\mathrm{C} 10$ & & $-\mathrm{C} 10$ & $123.8(2)$ \\
\hline $\mathrm{c} 6 \mathrm{a}$ & $a--\mathrm{C} 10$ & a--- & $\mathrm{C} 10$ & $117.7(2)$ & & & & & & \\
\hline
\end{tabular}

Version 1.6", 1993, Molecular Structure Corporation, Texas, U.S.A.

2. M. C. Burla, M. Camalli, G. Cascarano, C. Giacovazzo, G. Polidori, R. Spagna, and D. Voterbo, J. Appl. Cryst., 1989, 22, 389.

3. C. K. Johnson, "ORTEP-II, A FORTRAN ThermalEllipsoid Plot Program”, Report ORNL-5138, 1976, Oak Ridge National Laboratory, Tennessee, U.S.A. 